\title{
Grinding Motor Energy Saving Method based on Material Removal Model in Rail Grinding Processes
}

\author{
Shaodan Zhi', Jianyong Li',", and Allan M. Zarembski \\ 1 School of Mechanical, Electric and Control Engineering, Beijing Jiaotong University, Beijing, China, 100044 \\ 2 Department of Civil and Environment Engineering, University of Delaware, Newark, DE, USA, 1971 \\ \# Corresponding Author / E-mail: jyli@bjtu.edu.cn, TEL: +86-10-516-83685, FAX: +86-10-516-83685
}

KEYWORDS: Rail grinding, Grain distribution, Grinding power, Cutting force, Cutting speed

\begin{abstract}
Railway is an environmental friendly method which has been widely used in the land transportation driven by electric powers, without harmful emissions and unhoped traffic jams. And it is important to guarantee the running safety through the technology of rail grinding. However, the empirical processes have limitations on the expanded requirements of frequent and systematic rail grinding. Therefore, it is necessary to build up the rail grinding model to fill the gap between the operating parameters and desired on-theground results. In this paper, a three-dimensional distribution model has been built to get a uniform special-distribution for grains and extract one slice as the representative grinding surface, with decahedrons grains transformed from spheres. Then the cutting force model and then related power model have been achieved based on the formed grinding surface with information of grains geometry and their locations. Calculation results show that the consumed power or the consumed energy have a close relationship with grinding speeds and grinding forces, which are closely related to the grain geometry, involved grain numbers, location of grains and their cutting depth. And it comes to a conclusion that a judgment for energy-saved grinding strategy depends on the grinding aims.
\end{abstract}

\section{NOMENCLATURE}

$\mathrm{S}=$ tense type of grinding wheel

$\mathrm{M}=$ grade of grain size

$d_{\text {ave }}=$ average diameter of grains

$v_{g}=$ volume percentage of grains in the grinding wheel

$l_{r}=$ average distance of adjacent grains

$N_{\text {cut }}=$ numbers of transversal circles on the cutting slice

$h_{z m}=$ z-coordinate of cutting slice

$M_{\text {slice }}=$ number of cutting slices

$s_{n}=$ transversal area of nth circle on the cutting slice

$S_{m}=$ interaction area sum of mth slice

$d_{i j k}=$ diameter of nth grain with ID ijk

$d_{i j k}^{n}=$ diameter of nth interaction circle with grain ID ijk

$z_{i j k}=\mathrm{z}$-coordination of grain with ID ijk

$\bar{S}=$ average of area sum

$\sigma_{\text {area }}=$ area variance of all cutting slices

$(x, y, z)=$ origin coordinate system $\left(x^{\prime}, y^{\prime}, z^{\prime}\right)=$ coordinate system after rotation

$\mathbf{M}_{\mathrm{x}}=$ transfer matrix of rotation around $\mathrm{x}$ axis

$\mathbf{M}_{\mathrm{y}}=$ transfer matrix of rotation around $\mathrm{y}$ axis

$\mathbf{M}_{\mathrm{z}}=$ transfer matrix of rotation around $\mathrm{z}$ axis

$\mathbf{P}_{m}^{o}=$ location of origin point in decahedron $(\mathrm{m}=1, \ldots, 12)$

$\mathbf{P}_{m}^{\mathrm{r}}=$ location of point after rotation $(\mathrm{m}=1, \ldots, 12)$

$\theta_{\mathrm{x}}=$ rotation angle around $\mathrm{x}$ axis

$\theta_{\mathrm{y}}=$ rotation angle around $\mathrm{y}$ axis

$\theta_{\mathrm{z}}=$ rotation angle around $\mathrm{z}$ axis

$h=$ undeformed chip thickness

$h_{c}=$ chip thickness

$\mathrm{F}=$ friction force on the tool-chip face

$\mathrm{N}=$ normal force on the cutting plane

$\mathrm{Fs}=$ shearing force on the shearing plane

$\mathrm{Fn}=$ normal force on the shearing plane

$\mathrm{Fs}=$ cutting force

$\mathrm{Ft}=$ thrust force

$\gamma=$ rake angle 

$\alpha=$ flank angle
$\beta=$ friction angle
$\varphi=$ shear angle
$\tau_{s}=$ shear stress
$\sigma_{s}=$ normal stress
$\mathrm{A}_{\mathrm{s}}=$ area of shear plane
$\vec{R}=$ radius distance of grain to the wheel center
$\vec{\omega}=$ rotation speed
$\vec{v}=$ forward passing speed of rail grinding train
$\vec{v}_{c}=$ resultant cutting speed
$\mathrm{p}_{i j k}=$ power consumed on individual grain with ID ijk
$\vec{F}_{c}^{i j k}=$ cutting force vector
$\vec{v}_{c}^{i j k}=$ cutting speed vector
$P_{\text {com }}=$ consumed power of all grains
$P_{\text {at_rotation }}=$ power spent at grinding wheel rotation
$P_{\text {at traction }}=$ power spent at traction of rail grinding train

\section{Introduction}

Railway transportation is a typical green way especially for the massive land carriage driven by electric powers, without harmful emissions and unhoped traffic jams. ${ }^{1}$ Another significant feature of rail transportation is the long service life of rails to support the vehicle traveling. Once the rail lines have been paved, the function of guiding trains can sustain permanently without necessary rail replacements. As one of the outstanding country with high speed railways, China has broken through 10,000 kilometers with the railway traffic across north and south with average speeds over 300 kilometers per hour. Such running status reveals a high density of operation which brings a high rate of deterioration on the track structures in general and on the running surface of the rails in particular. Moreover, these wears of the running surfaces such as corrugations and fatigue cracks will develop into surface as well as internal defects, which impact the running comfort of the trains and even threaten the running safety.

An engineering method for removing these defects is rail grinding. Such advanced technique has been adopted worldwide to eliminate surface damage and reposition contact points between the wheel and the rail. ${ }^{2}$ Eventually, rail grinding is developed from grinding machine into rail grinding trains with dozens of grinding motors to catch up with the requiring grinding efficienc. ${ }^{3}$ Consequently, the rail grinding can achieve long term benefits for railway transportation through removing the early levels of defects ahead of endangering the running conditions of railway vehicles and compelled rail replacement.

To sum up, rail grinding becomes a green manufacturing technology by achieving the followings:

- Rail grinding strengthens the efficiency of green railway transportation with guarantees of safety running surfaces with minimum disturbance on traffic.

- Rail grinding prolongs the rail life and reduces the demands of steel production especially for replacing worn rails, with the follow-on reduction of industrial pollution.

- A fine running environment which are maintained by rail grinding can reduce the power consumption on traction during the vehicle travelling.

As time goes on, more attentions are paid on the rail grinding efficiency for the busier schedules of modern railway networks with reduced time windows for maintenance. Specifically, the rail grinding operations need to increase the grinding speed while keeping with the necessary metal removal. In order to achieve this, a better understanding of rail grinding needs to be developed based on the key parameters of the rail grinding process and associated factors in the rail grinding process.

Generally speaking, however, researches which only rely on the experiences of grinding projects are limited to being applied to similar rail operations and may not be optimal for the current operation. Furthermore, it takes months or even longer to understand what needs to be ground and how effective the grinding is. Moreover, these grinding parameters may not be suitable to other rail line because of the different traffics, track conditions and rail defects. For example, the planning of rail grinding needs to address the crack growth in a specific period of time so as to remove incipient cracks without being overconservative and producing excessive material loss. ${ }^{4}$

It can be said that the empirical process for exploring the optimal grinding strategy for a single rail line or for the whole rail networks cannot adapt to the expanded development of high-speed railways and busy rail networks which call for an optimal maintenance strategy with frequent, efficient and systematic rail grinding. The concepts for optimizing rail grinding process could not be achieved unless more scientific and systematic researches are explored for the relationships between the grinding parameters and the grinding results. Further development also includes the concept of life-cycle-cost (LCC) for preventive grinding to achieve maximum rail life with minimum cost based on optimal rail grinding activities. ${ }^{5}$

All in all, the further development of rail grinding investigations needs to progress from the macroscopic general-strategy-level into the explicit-factor-level so as to define and select the essential rail grinding capabilities and characteristics. Then it will be possible to fill the gap between the operating parameters and desired on-the-ground results of rail grinding. With further step into the rail grinding mechanism, the rail grinding wheels are the manufacturing tools which have vary grinding capabilities at different grinding speeds, different types/compositions. And the complicated interactions between the grinding wheels and the rails are the entrances for a systematic theoretical research of rail grinding.

In this paper, the model of the grinding wheel and its capability of consuming power are built based on the random distribution of cutting grains and their cutting mechanism to form a proper description of the grinding process.

First of all, the sphere grains are located with equal distance as the initial status to simulate the three-dimensional realistic distribution model of the grains in the grinding wheel. Then each grain is given stochastic micro-displacements without intervening into any others during each "shaking" operation for the random distribution. The uniform random-distribution of grains is obtained after thousands of shaking times through a measuring standard with cross-section areas of grains on equal-interval slices, as in Section 2. Secondly, one slice is chosen from the spatial model to represent the grinding surface of the 
grinding wheel. To step forward of simulating the arbitrary shapes of grains as in the realistic grinding wheel, the sphere grains of this chosen slice are changed into arbitrary polyhedron grains with arbitrary poses to get obtuse cutting angles of grains, as in Section 3. Thirdly, the cutting model of individual grain established based on the cutting mechanics. Moreover, the power consumption are carried out with consideration of grinding speeds which are resultants of the rotating speeds of the grinding wheel and the forward speeds of the rail grinding train, as in Section 4.

\section{Three-Dimensional Model of Uniform Distribution Grains for Grinding Surface}

\subsection{Initial Preparation with Equal Spaced Grains}

For the case of rail grinding, based on the specifications of rail grinding wheel chosen for the rail grinding trains, the corresponding tense type is $S=14$ and the grain size is $M=36$. According to Malkin's research, the grain size of $M$, the average diameter for cutting grains, the volume of grains and the average distance of adjacent grains can be obtained through Equ. 1-3 as followings: ${ }^{6}$

$$
d_{\text {ave }}=68 \cdot M^{-1.4}
$$

where $d_{\text {ave }}$ is the average diameter of the grains.

The volume percentage of grains in the grinding wheel can be estimated.

$$
v_{g}=2 \cdot(32-S) / 100
$$

The average distance between the adjacent grains is:

$$
l_{r}=d_{\text {ave }} \cdot \sqrt{0.25 \cdot \pi / v_{g}-1}
$$

The grain centers are supposed to be arranged with equal interval distance $\left(l_{r}+d_{\text {ave }}\right)$ between the grain centers before the shaking operation. The grains which belong to different quadrants are marked with different colors, as shown in Fig. 1. The average diameters of the grains, however, are not implemented into the simulation progress.

Accordingly, the sizes of the grains have varying diameters by following a normal distribution, with sizes as shown in Fig. 2.

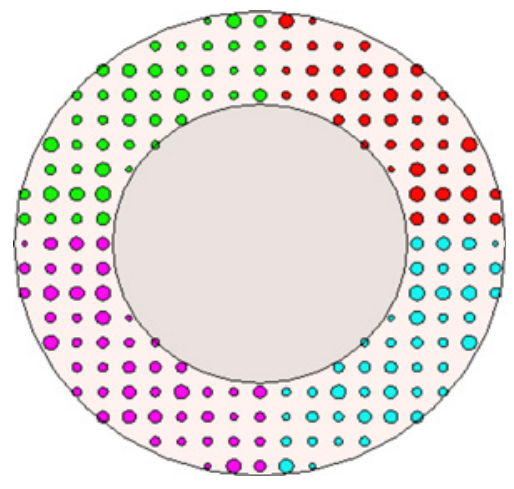

Fig. 1 Schematic diagram for equally spaced grains

\subsection{Shaking Simulation}

The method of shaking grains in the whole model is implemented by moving the grains sequentially without interacting any of the others. Besides, the grains are moving sequentially without running out the boundary. Based on the equal spaced distribution in the whole geometry model of grinding wheel, the amount of the grains can be finalized with required information as shown in Fig. 3. The grains in the geometry model need to be recognizable before any shaking (moving) happens. Therefore, identified serial numbers (ID) are given to the overall grains one by one, with assigned diameter sizes.

Since every grain is moving freely in the whole model, the locations of the grains and their relative positions are changing all the time along with the displacement of each grain. During one time of shaking process, each grain is given with a random displacement in the threedimensional boundary in the wheel space. If this grain intervenes into any other grains or runs out of the wheel space, another random displacement is then applied to this grain until none of the situations mentioned above happen, as shown in Fig. 4.

Once the moving grain is settled with an allowable new location, then the next grain is on the process. Only with all grains are moved once, one shaking can be regarded as completed. Then repeat this shaking process over and over again until a stable random distribution is achieved. This method is rather time consuming since it requires checking the

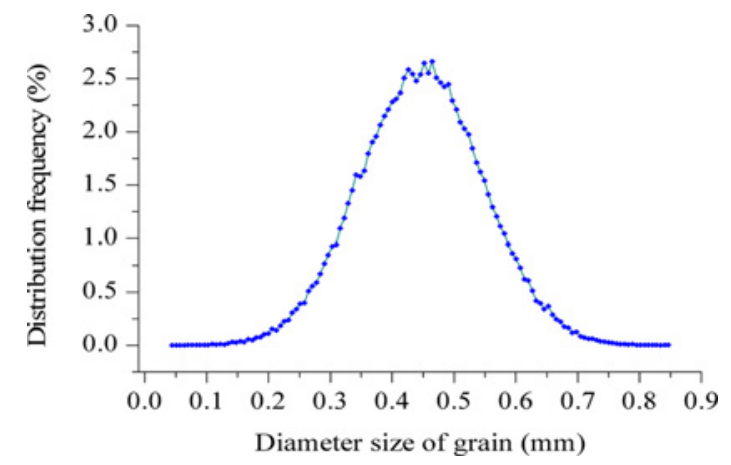

Fig. 2 Distribution frequency of diameter size

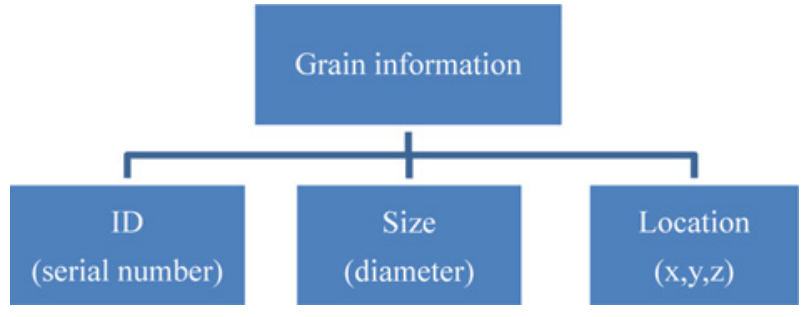

Fig. 3 Necessary information for each grain

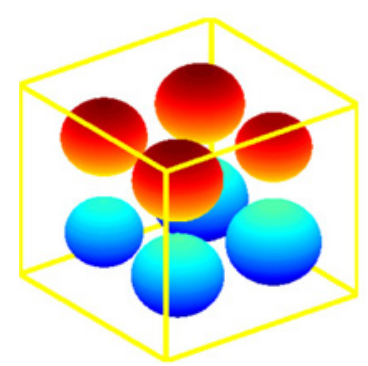

Fig. 4 Illustration of grains and boundary 
interaction between the displaced (moved) grain and all others in the model using random displacement for each grain in each shaking.

\subsection{Uniform Distribution}

In order to investigate the uniformity of the grains distribution after several shaking cycles, cutting "slices" with equal intervals are set-up to interact with grains and sum the cross sectional areas of the interaction circles on the same slice. As shown in Fig. 5, the cutting slice plane interacts with the grains and forms corresponding circles in this slice plane with the sizes of the grains pitch-diameters. Once the grains are removed in the three-dimensional space, cross-section circles left on the same slice plane will be changed for their changed relative position toward the slice plane.

Since one slice can represent the positions of the grains which interact into the slice plane, plenty of slices can be used to get the $3 \mathrm{D}$ space distribution by being arranged in equal distance. The variance for the accumulated areas of circles on different slices can be taken as the measurement of the uniformity of grain distribution. The cutting plane is horizontal with z-coordinate of $h_{z m}\left(1 \leq \mathrm{m} \leq M_{\text {slice }}\right)$, where $M_{\text {slice }}$ represents the number of cutting slices with equal intervals. Then the numbers of transversal circles on the slice can be obtained by checking the distance from the grain center to the slice plane:

$$
N_{c u t}=\sum \text { find }\left(\left|z_{i j k}-h_{z m}\right|<0.5 \cdot d_{i j k}\right)
$$

For the $m$ th slice, the sum of interaction area $S_{m}$ can be obtained by summing up all the interaction circles founded:

$$
\left\{\begin{array}{c}
S_{m}=\sum_{n=1}^{N_{\text {cut }}} s_{n} \quad\left(1 \leq m \leq M_{\text {slice }}\right) \\
s_{n}=\pi \cdot\left[\left(0.5 \cdot d_{i j k}^{n}\right)^{2}-\left|z_{i j k}^{n}-h_{z m}\right|^{2}\right]
\end{array}\right.
$$

Where $s_{n}$ is the area of the $n$th interaction circle; $d_{i j k}^{n}$ represents the diameter of $n$th interaction circle which can be traced by the grain ID (ijk) to get from the original data of grain.

The average area-sum for all cutting slices would be

$$
\bar{S}=\frac{1}{M_{\text {slice }}} \cdot \sum_{m=1}^{M_{\text {slice }}} S_{m}
$$

Then the area variance of all cutting slices can be calculated to estimate the distribution results for one shaking process:

$$
\sigma_{\text {area }}=\sqrt{\frac{1}{M_{\text {slice }}} \sum_{m=1}^{M_{\text {slice }}}\left(S_{m}-\bar{S}\right)^{2}}
$$

The distribution model reaches its variance limitations of after 5000

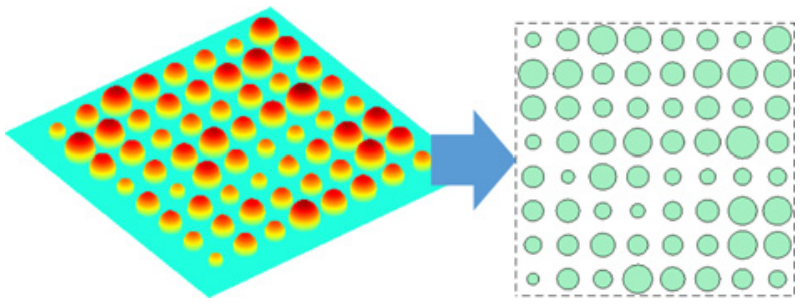

Fig. 5 Cutting slice and transversal circles times of shaking. That means, even with the increase of shaking times, the area variance of cutting slices cannot be decreased. The distribution of grains in such three dimensional spaces are almost immobilized. Therefore, such status indicates a uniform distribution of those grains.

The area-sums of circles on different slices are recorded before and after 5000 shaking cycles. The contrast results of area-sums, as shown in Fig. 6, fluctuate in an analogous-sine way in the original model before shaking. After 5000 times of shaking to an immobilized uniform status, the area-sums curve flattens out. The amplitude of this analogous-sine wave is almost $200 \mathrm{~mm}^{2}$ at the start of the shaking. After 5000 shaking cycles to the movement limitation, the largest fluctuation of the area-sums for these slices is less than $20 \mathrm{~mm}^{2}$, less than $10 \%$ of the former amplitude. The local flat section in the curve after shaking indicates a uniform distribution of the grains. A completely flat curve could happen only if all the grains have the same diameters. Therefore, some fluctuation is inevitable with diameters of grains complying with normal distribution so as to simulate the actual situation of grains in the grinding wheel.

\section{Extraction of Grinding Surface}

A volumetric uniform distribution is eventually accomplished after the shaking operation as mentioned above. Since the direct interaction between the grinding wheel and the rail happens on their surface, it is necessary to extract the representative grinding surface to analyze the grinding process. ${ }^{7,8}$ One cutting slice which has the most flattened areasums of circles are chose as the representative grinding surface from Fig. 6. Among those grains protruding over the slice plane, nearly half of grains have their centers concealed beneath, as shown in Fig. 7.

Since there will be cutting moments formed on the top of the grains caused by the cutting forces, the cutting stability will be affected for the grains with centers above the slice plane. They are more likely to have the cutting moments large enough to overturn themselves. Therefore,

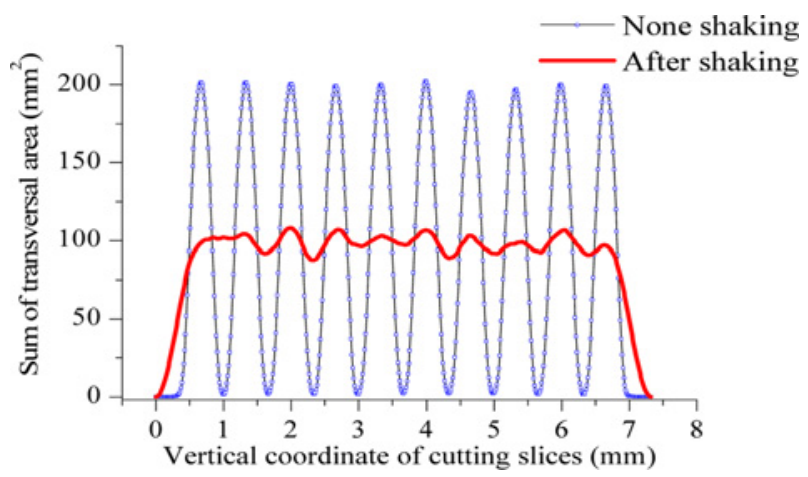

Fig. 6 Area-sums of slices before and after shaking

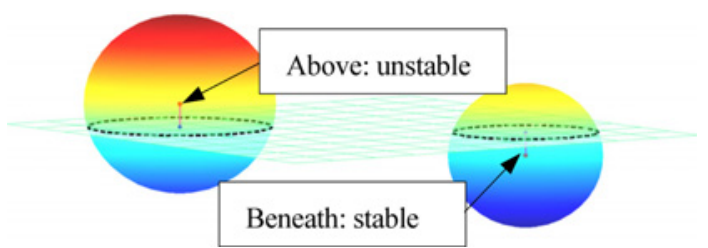

Fig. 7 Identification of stable and unstable grains 
the grains with centers above the slice are assumed to be unstable. The grains with centers beneath are taken as the providers of the active cutting edges for the stable cutting states.

After deleting the unstable grains, the numbers of suitable active grains are accounted for according to their potential cutting depth (based on protrusion heights). There are thousands of grains on the grinding surface though, only a minority of grains plough into the rail surface when the cutting depth is set at a very small value. That is to say, the grain with highest protrusion has the first priority to touch the rail surface. Once the cutting depth is increased, more grains will join into the group of active cutting grains to carry on the grinding process, as shown in Fig. 8.

The spherical shape for the grains proposed was very helpful to generate the spatial grain distribution. However, the ideal shape is not necessarily spherical in the actual grinding process but arbitrary shapes. The further simulation of the grain's cutting mechanism requires a transformation of grain shapes by replacing the sphere grains with arbitrary polyhedron grains. As shown in Fig. 9 (a), an arbitrary hexahedron can be carved out of the sphere grain. This is a rough treatment to imitate the surface of the grinding wheel.

If an arbitrary hexahedron is generated to replace the spherical grain, the angles between the potential cutting edge and the cutting plane will be possible sharp other than blunt. The residual between the simulation and the actual status will be increased if the grinding wheel surface is glutted with too many sharp angles. As a result, an arbitrary decahedron is more applicative to be generated in the cutting grain. As shown in Fig. 9 (b), an arbitrary decahedron has a closer protrusion height and a closer volume to the original sphere grain than a hexahedron. Rather than keeping the static poses, the arbitrary decahedron grains need to be rotated at random angles to get closer to the arbitrarily complex grinding wheel surface. More importantly, the blunt cutting angles can be promised with whichever

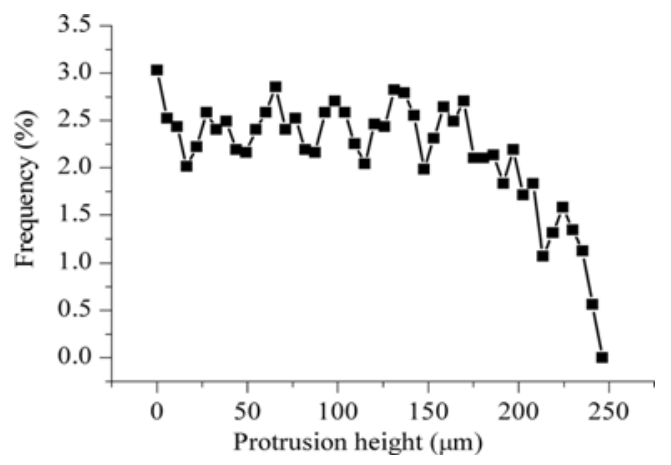

Fig. 8 Frequency of active grains at different protrusion heights

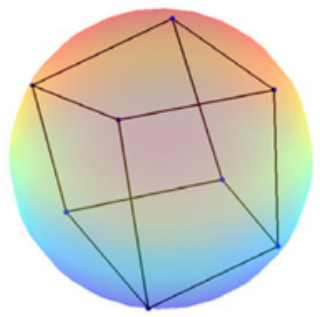

(a)

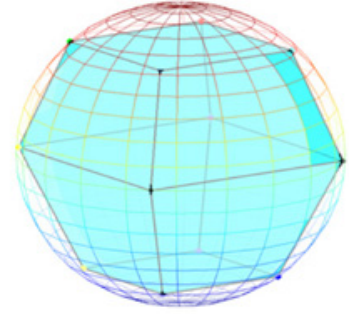

(b)
Fig. 9 Diagram of hexahedron and decahedron transformations edge become the cutting edge. And the way that determines the cutting edge lies in the arbitrary rotations around the axes in the threedimensional coordinate system. As shown in Fig. 10 (a), the origin decahedron grain without any rotation is exhibited with coordinate system $(x, y, z)$ locates in its center. Such coordinate system is taken as the local coordinate system which rotates with the decahedron grain and reflects the poses of the grain. And the golobal system is the origin system without rotation to record any changed positions.

Supposing that the grain is rotated around $\mathrm{x}$ axis with angle $\theta_{\mathrm{x}}$, as shown in Fig. 10 (b), the local coordinate system is rotated as well to the new position at $\left(x^{\prime}, y^{\prime}, z^{\prime}\right)$. It's found that the $\mathrm{x}$ axis is coincident with new one $x^{\prime}$. Since the distortion need to be prevented, the points of the decahedron are not moved to the new coordinate $\left(x^{\prime}, y^{\prime}, z^{\prime}\right)$. This is to say, the coordinate values denoted with the new coordinate are remained. Therefore, the transfer matrix for the rotation around $\mathrm{x}$ axis would be

$$
\mathbf{M}_{x}=\left[\begin{array}{ccc}
1 & 0 & 0 \\
0 & \cos \theta_{x} & \sin \theta_{x} \\
0 & -\sin \theta & \cos \theta_{x}
\end{array}\right]
$$

And the relationship between the locations of the rotated points and the origin ones would be

$$
\mathbf{P}_{m}^{\mathrm{r}}=\mathbf{P}_{m}^{\mathrm{o}} \cdot \mathbf{M}_{x} \quad(m=1, \ldots, 12)
$$

And by this anology, the rotation angles around $\mathrm{y}$ axis and $\mathrm{z}$ axis are supposed to $\theta_{\mathrm{y}}$ and $\theta_{\mathrm{z}}$, as shown in Fig. 10 (b)-(c). Then the transfer matrixes would be

$$
\mathbf{M}_{y}=\left[\begin{array}{ccc}
\cos \theta_{y} & 0 & -\sin \theta_{y} \\
0 & 1 & 0 \\
\sin \theta_{y} & 0 & \cos \theta_{y}
\end{array}\right] \quad \mathbf{M}_{z}=\left[\begin{array}{ccc}
\cos \theta_{z} & \sin \theta_{z} & 0 \\
-\sin \theta_{z} \cos \theta_{z} & 0 \\
0 & 0 & 1
\end{array}\right]
$$

And the locations of the points on the decahedron after the rotations are obtained through these transfer matrixes as followings:

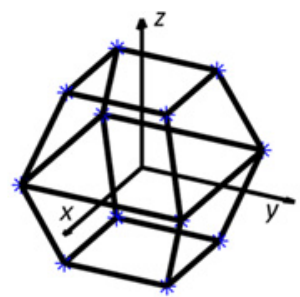

(a) None rotation

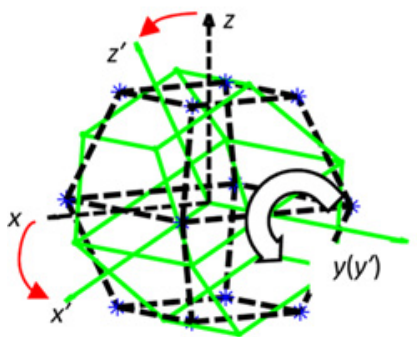

(c) Rotate around y axis

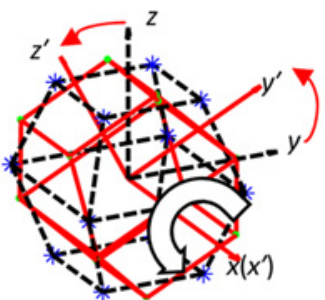

(b) Rotate around $x$ axis

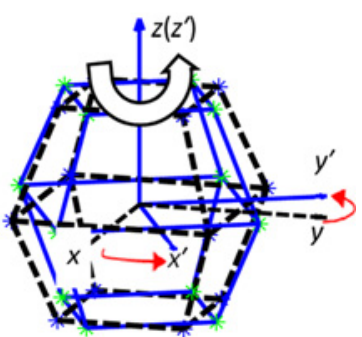

(d) Rotate around $\mathrm{z}$ axis
Fig. 10 Illustration of none-rotation and rotations 


$$
\begin{aligned}
& \mathbf{P}_{m}^{\mathrm{r}}=\mathbf{P}_{m}^{\mathrm{o}} \cdot \mathbf{M}_{y} \quad(m=1, \ldots, 12) \\
& \mathbf{P}_{m}^{\mathrm{r}}=\mathbf{P}_{m}^{\mathrm{o}} \cdot \mathbf{M}_{z}
\end{aligned}
$$

By bridging the connections between the rotating operation and the point locations of the decahedron grain, it is convenient to see that these transfer matrixes are independent. Eventually, new locations will be found with arbitrary rotation angles $\left(\theta_{\mathrm{y}}, \theta_{\mathrm{y}}, \theta_{\mathrm{z}}\right)$.

$$
\mathbf{P}_{m}^{\mathrm{r}}=\mathbf{P}_{m}^{\mathrm{o}} \cdot \mathbf{M}_{\mathrm{x}} \cdot \mathbf{M}_{\mathrm{y}} \cdot \mathbf{M}_{\mathrm{z}} \quad(m=1, \ldots, 12)
$$

By following the rotation method as mentioned above to rotate the grains one by one with arbitrary angles, the surface grains are replaced eventually by the arbitrary decahedron ones with random rotating angles, as shown in Fig. 11. In such a way, a grinding surface with a number of widespread blunt cutting edges can be built up to have a step closer to the complexity of the realistic grinding surface.

\section{Grinding Powers based on Grain Cutting}

\subsection{Cutting Model of Single Grain}

The key to determining the cutting depth achieved by each abrasive grain is the analysis of the cutting process and the resulting metal sliver or "chip" that is removed by that grain or grit. According to basic machining theory. The cutting progress for each individual cutting grain that occurs during grinding can be illustrated as shown in Fig. 12. As one chip is formed, the size of which is dependent on the geometry of the chip and the work piece (the rail).

This geometry is defined by a series of three planes; the rake face (with rake angle $\gamma$ ), the flank face (with the flank angle $\alpha$ ) and shear plane (with the shear angle $\varphi$ ). At a given cutting speed, the removed chip moves away from the rake face with a corresponding speed referred to as the chip velocity. The removed chip will have a chip thickness $h_{c}$, while the remaining portion will have the undeformed chip thickness $h$,

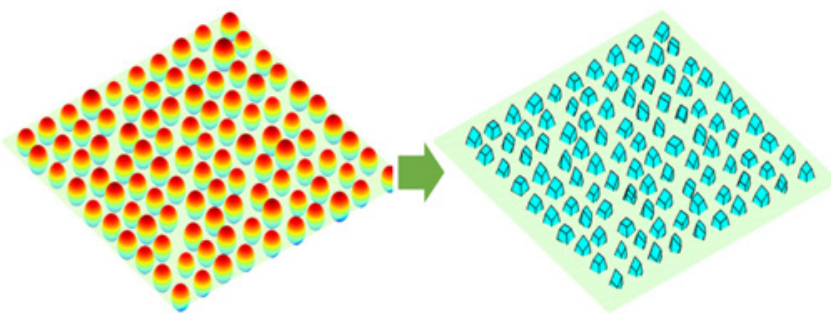

Fig. 11 Shape transformation on surficial grains

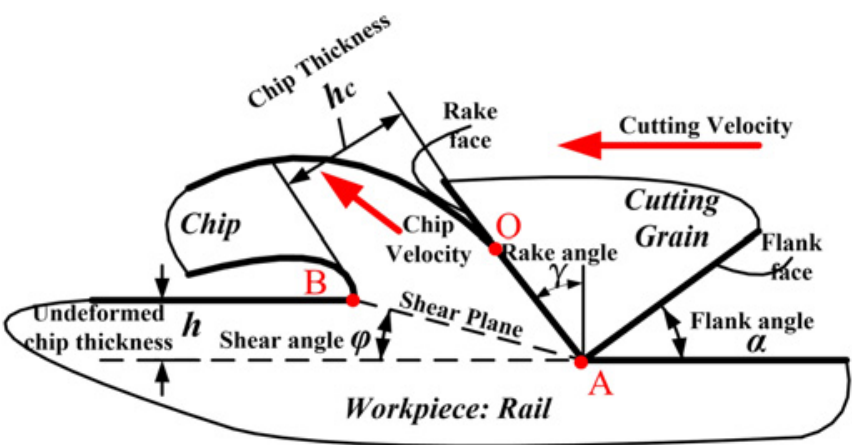

Fig. 12 Chip formation model for single cutting grain which represents the cutting depth of the individual grain or grain.

Based on the theory of force equilibrium by Merchant, as seen in Fig. 13, the deformed chip is subjected to two opposing forces $T$ and $T^{\prime}$, which are the active force and the passive force, respectively. These two forces can be defined as the resultant forces at the shear plane and rake face.

The force $T$, which the tool exerts on the chip, is resolved into the tool face-chip friction force $F$ and normal force $N$. The angle $\beta$ between $F$ and $N$ is thus the friction angle. The force $T$ ' which the workpiece exerts on the chip is resolved along the shear plane into the shearing force $F_{s}$, which is responsible for the work expended in shearing the metal, and into the normal force, $F_{n}$, which exerts compressive stress on the shear plane. Force $T^{\prime}$ 'is also resolved along the direction of tool motion into $F_{c}$, termed as the cutting force, and into $F_{t}$, the thrust force.

The relationship between these forces can be obtained through the cutting geometry as followings:

$$
\begin{array}{r}
\left\{\begin{array}{l}
F_{s}=T \cdot \cos (\varphi+\gamma+\beta) \\
F_{n}=T \cdot \sin (\varphi+\gamma+\beta)
\end{array}\right. \\
\left\{\begin{array}{l}
F_{c}=T \cdot \cos (\gamma+\beta) \\
F_{t}=T \cdot \sin (\gamma+\beta)
\end{array}\right.
\end{array}
$$

Though the forces as mentioned above make up the equilibrium, the most concerned force is the cutting force $F_{c}$, which can be expressed as:

$$
F_{c}=\frac{\cos (\gamma+\beta)}{\cos (\varphi+\gamma+\beta)} F_{s}
$$

Noting that the concepts of shear stress and normal stress are directly addressed for the shear plane $A_{s}$. The normal force on the shear plane $F_{n}$ brings along the normal stress $\sigma_{s}$ and the shear force $F_{s}$ induces the shear stress $\tau_{s}$ in the rail material. According to the rail hardness and strengths related to the analyses, ${ }^{4}$ the relationship between the shear stress $\tau_{s}$ and normal stress $\sigma_{s}$ can be found to be:

$$
\tau_{s}=F_{s} / A_{s}, \tau_{s} \approx 0.45 \cdot \sigma_{s}
$$

Then the shear force can be obtained through the geometry information such as plane area $A_{s}$ and the material properties of workpiece:

$$
F_{s}=A_{s} \cdot \tau_{s} \approx 0.45 \cdot A_{s} \cdot \sigma_{s}
$$

Then the cutting force generated on the cutting grain would be closely related to the geometry of the cutting grain:

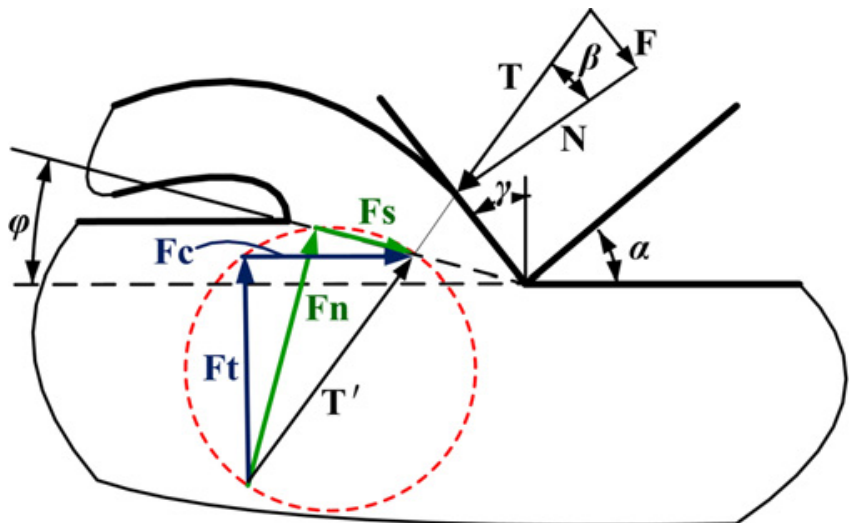

Fig. 13 Cutting force analysis of cutting grain 


$$
F_{c}=\frac{\cos (\gamma+\beta) \cdot 0.45 \cdot \sigma_{s}}{\cos (\varphi+\gamma+\beta)} \cdot A_{s}
$$

\subsection{Cutting Speeds}

Since the cutting grains are randomly distributed on the grinding surface which are obtained as through the shaking model of the grinding wheel, the cutting speeds on the forming chips are varying based on the locations of these cutting grains. As shown in Fig. 14, 20 grains with different locations on the wheel are chosen to present the vectors of the rotation speeds and the forward speeds. The tangential velocities which marked by red arrows actually are the linearized rotation speed $w R$. Therefore, it is worthy of attention that even with the same angular speed, the linearized rotation will be different if the grains are located at different distance to the wheel center, i.e., $R$ varies according to the grain's position $(75 \mathrm{~mm} \leq R \leq 125 \mathrm{~mm})$. The rotation angular speed of the grinding wheel is kept at $w$, while the forward speed $v$ which represents the changeable forward passing speed of the rail grinding train. When the window time for the grinding maintenance is limited, the forward passing speed of the rail grinding train needs to be increased which will make the resultant velocity changes as well.

Since the cutting speed is the relative speed exists between the grain and the rail, it is necessary to combine these two kinds of vectors as into the cutting speed $\vec{v}_{c}$ for individual grains.

$$
\overrightarrow{v_{c}}=\vec{\omega} \times \vec{R}+\vec{v}
$$

The resultant operation needs removing these two vectors to the same start point, as shown in Fig. 15.

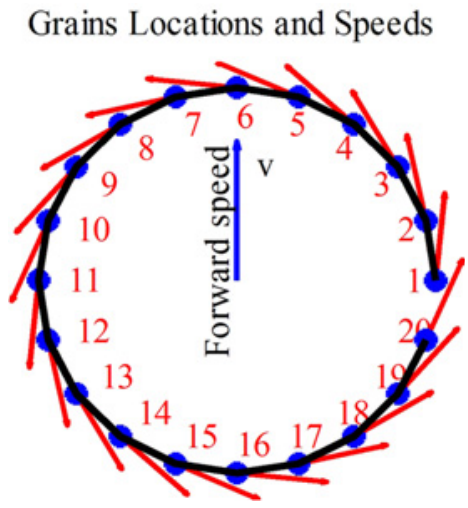

Fig. 14 Vectors for forward speed and rotate speed

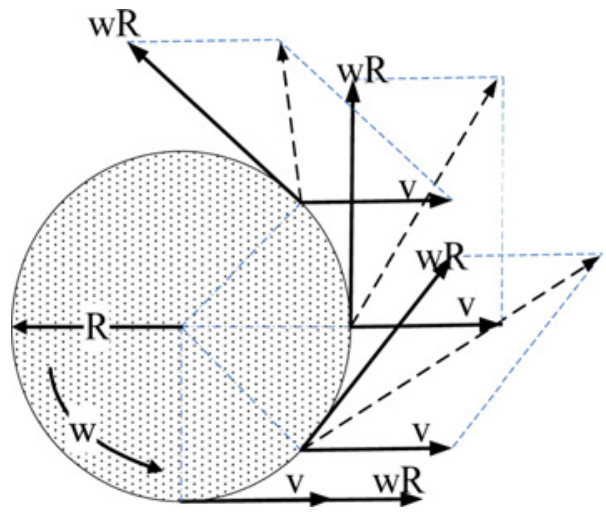

Fig. 15 Schematic diagram of resultant operation
By putting all the velocity vectors into one start point, the resultant velocities results for the grains with same distance to the wheel center can be analyzed. As shown in Fig. 16 (a)-(d), the red dashed lines represent the rotation vectors, and blue lines are the respective resultant velocities. It can be noticed that with the improving of the forward speed from $4 \mathrm{~km} / \mathrm{h}$ to $64 \mathrm{~km} / \mathrm{h}$, the resultant vectors are increased at the positive direction with the increased forward speed. And if the forward speed is large enough, the resultant velocities will have no negative vectors against the forward speed.
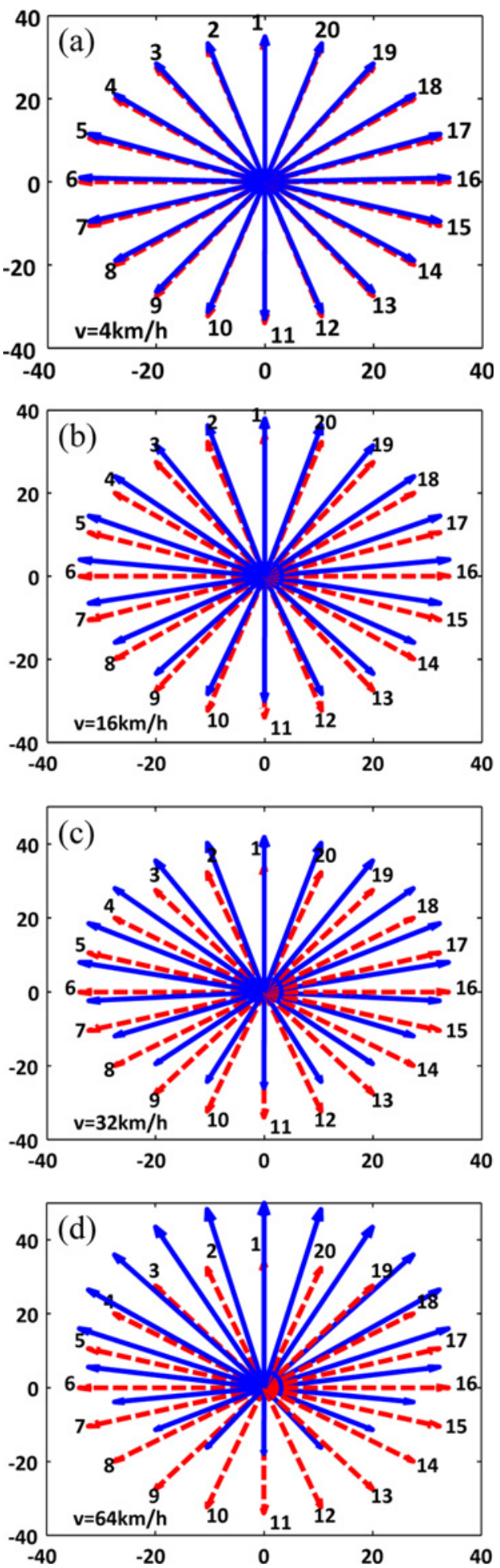

Fig. 16 Resultant velocity with variable forward speed 


\subsection{Power Consumption of Rail Grinding}

Based on the analyzing on the cutting behavior, it can be derived that the cutting force vector and the cutting velocity vector are at the same direction for the same individual cutting grain. ${ }^{9}$ Therefore, the power consumed for the individual grain (with recognized ID ijk) which are involved into the grinding process can be obtained through dot-product of the cutting force vector and the cutting velocity vector.

$$
p_{i j k}=\vec{F}_{c}^{i j k} \cdot \vec{v}_{c}^{i j k}
$$

And the power consumed in the grinding process for one grinding wheel can be obtained by summing up all the consumption from all involved grains:

$$
P_{c o m}=\sum p_{i j k}=\sum \vec{F}_{c}^{i j k} \cdot \vec{v}_{c}^{i j k}
$$

In the rail grinding process, it can be deemed that the consumed power for rail grinding comes from two ways: grinding motors which drives the rotation of the grinding wheel and the forward traction which drives the forward running of the rail grinding train. For the convenience of analyzing, the power comes from these two ways are calculated from the productions of the component grinding forces along the direction of the rotation velocity as well as the forward speed, which signify the dot production of the cutting forces and the velocities, respectively.

$$
\begin{gathered}
P_{\__{\text {at_rotation }}}=\sum \vec{F}_{c}^{i j k} \cdot\left(\vec{\omega}^{i j k} \times \vec{R}^{i j k}\right) \\
P_{-a t+t r a c t i o n}=\sum \vec{F}_{c}^{i j k} \cdot \vec{v}^{i j k}
\end{gathered}
$$

\subsection{Calculation and Discussion}

The calculations are carried out based on the analyzing equations as shown above. By combing the models of the cutting forces and cutting velocities for individual cutting grains, the overall cutting efficiency can be reflected on the consumed powers and consumed time. Ahead of this step of power calculation, the completed simulation for random distribution of surficial grains provides the protrusion heights which have key links to the grinding depths and the numbers of involved cutting grains. As shown in Fig. 8, the deeper the grinding wheel goes into the rail surface, the more grains will join into the cutting. Different grinding pressures are assumed to have relationships with the cutting depth: greater pressure matches with more grains.

For the calculation under a constant pressure, the number of involved grains into the cutting process is assumed to $90 \%$ of the surficial grains which protrude above the grinding surface of the rail grinding wheel. In another word, if there are $90 \%$ surficial grains are joining into the cutting, it will mean that the grinding wheel is severely pushed on the rail. Moreover, three different pressures are set with Pressure $1<$ Pressure $2<$ Pressure 3 . Hence three levels of grain numbers are proposed, with $13 \%, 34 \%$ and $60 \%$ grains involved, respectively.

\subsubsection{Calculation under Constant Pressure}

The first part calculations are carried out by setting the grinding wheel against on the rail surface with constant pressure (with $90 \%$ surficial grains involved). In such a way, the cutting depth of the individual grains and especially the involved cutting grains can be estimated so as to highlight the effects of the grinding speeds. From the calculation based on the Equ. 21, the power consumed driven by the grinding motor for the grinding wheel fluctuates over the grinding time, as shown in Fig. 17.

And the forward speed has a significant influence on the power fluctuation from the grinding motor. The higher the forward speed is (from $4 \mathrm{~km} / \mathrm{h}$ to $64 \mathrm{~km} / \mathrm{h}$ ), the greater the amplitude of the power curve will be. The power fluctuation increases the instant load on the grinding motor, thus repeated fluctuation will impact the service life of the grinding wheel. And another noticeable mutual trait for these curves is that they share one oscillation center which probably caused by the constant rotating speed for the rail grinding wheel.

The powers on the traction of rail grinding train are also calculated based on the Equ. 22. As shown in Fig. 18, the powers consumed on the forward direction will be increased proportionally as increasing the forward speed. There are also fluctuations existing in the power curves for direction of the rail grinding traction. And it also obeys the previous forecast trend that the requisite cutting power will be increased with the constant cutting depth but increased cutting speed.

Therefore, the combined results for consumed powers in the cutting process of rail grinding can be carried out based on Equ. 20. As shown in Fig. 19, the combined powers not only fluctuate over the grinding time, but also have growing oscillation center with the increase of forward speed.

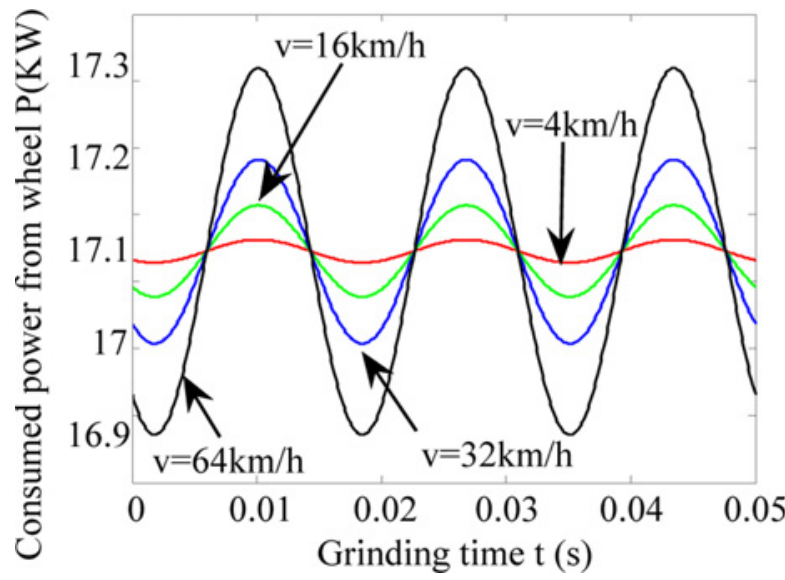

Fig. 17 Power consumption on wheel rotation

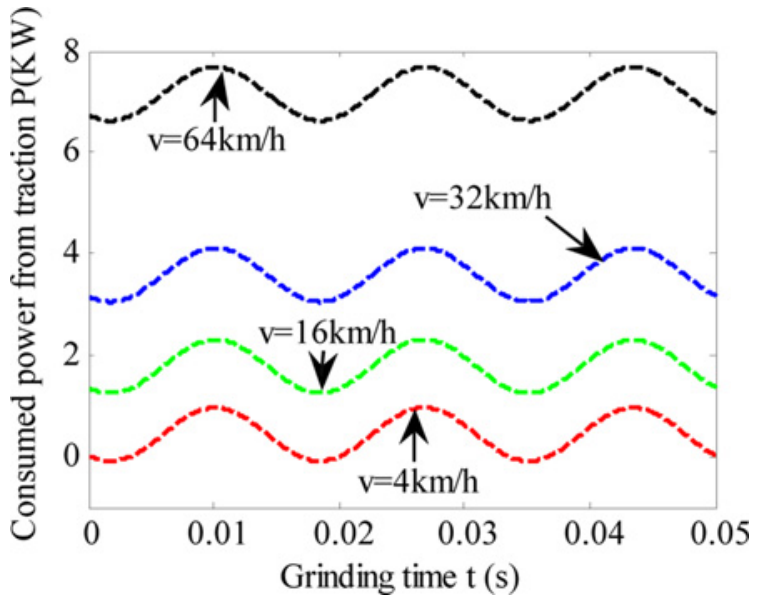

Fig. 18 Power consumption from traction 


\subsubsection{Calculation under Variable Pressures}

The results as above are carried out by setting a constant grinding pressure on the rail grinding wheel toward the rail surface. According to the protrusion heights of the grain distribution as in Fig. 8, the more cutting depths the grinding wheel achieve, the more grains will be involved into the cutting. Therefore, in this part, three successive incremental pressures are imposed on the rail grinding wheel, with $13 \%, 34 \%$ and $60 \%$ grains involved, respectively.

As shown in Fig. 20, three curved surfaces are the consumed power with the changes of grinding forward speed and time. The asterisks marked on the $\mathrm{z}$ axis are the start values of the consumed powers under different pressures. The power consumed under Pressure1 begins with $5.89 \mathrm{KW}$, and when the forward speed is increased to $64 \mathrm{~km} / \mathrm{h}$, the power will be up to $6.23 \mathrm{KW}$ (with increment of $0.34 \mathrm{KW}$ ). Under Pressure2, the consumed power begins with $32.55 \mathrm{KW}$, and when the forward speed is increased to $64 \mathrm{~km} / \mathrm{h}$, the power will be $34.21 \mathrm{KW}$ (with increment of $1.66 \mathrm{KW}$ ). For the third one, the consumed power begins with $92.57 \mathrm{KW}$, and when the forward speed is increased to $64 \mathrm{~km} / \mathrm{h}$, the power will be $97.24 \mathrm{KW}$ (with increment of $4.67 \mathrm{KW}$ ). The increasing on the forward speed result in different increments under different pressures: the more pressure is, the larger increments on consumed power from $4 \mathrm{~km} / \mathrm{h}$ to $64 \mathrm{~km} / \mathrm{h}$.

The same phenomenon of fluctuation happens for the same speed under the same pressure over the grinding time, as shown in Fig. 20. And the amplitude of such fluctuation increases with the increasing of

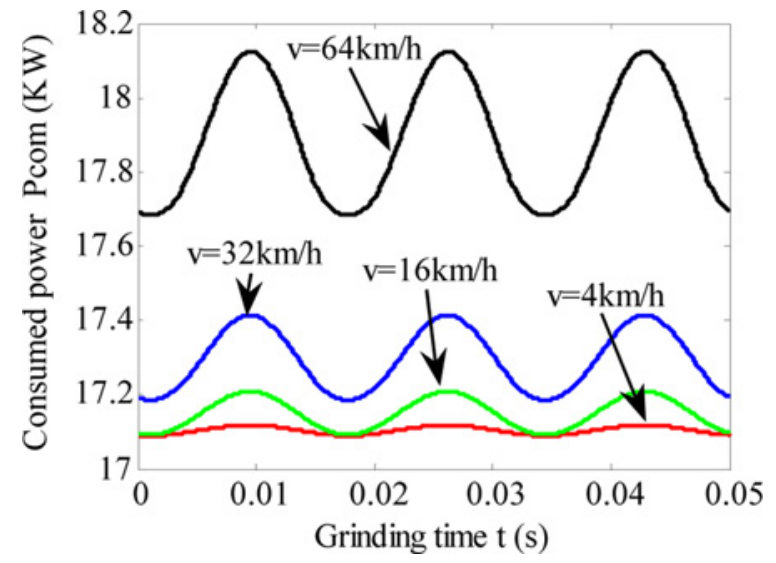

Fig. 19 Total power on rotation and forward running

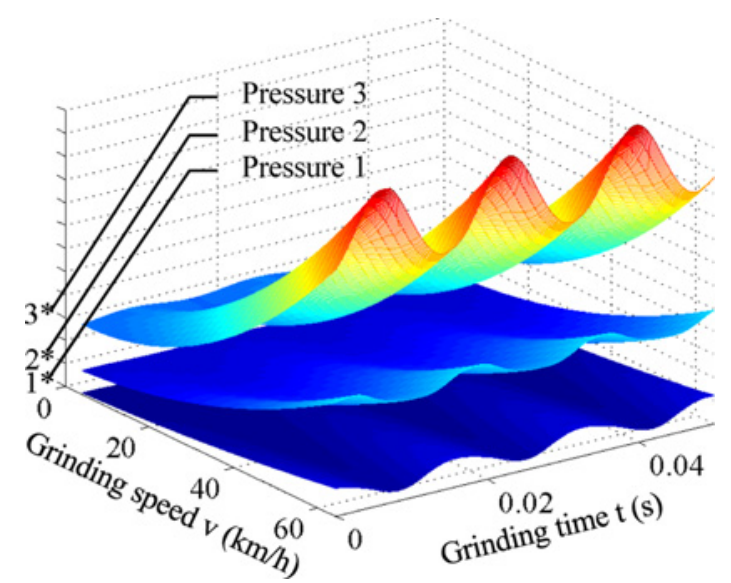

Fig. 20 Detail power consumption on different pressures forward speed and the pressure. Thus for the convenience of analyzing, these power surfaces are removed together in spite of the wide differences on the start value and incremental amplitudes. In this way, the fluctuation on surfaces can be also displayed.

After one time of grinding passing, average grinding depths can be achieved as shown in Fig. 21. The average grinding depths increase along with the increasing of pressures but decrease with the increased forward speed. It can be naturally concluded that the higher speed will lead to shorter times for grains to cut the rail, and higher pressure helps the grains cut deeper into the rail and get more material removed. Therefore, in order to achieve one same grinding task, the grinding running with lower average depth needs more running passes.

With the same grinding assignments on $1 \mathrm{~m}$ length rail, it is necessary for the lower-pressure grinding and the higher-speed grinding to grind the rail repeatedly until the grinding removals are achieved. Combining Fig. 21 and Fig. 22, it is clear that the consumed energies for the same metal removal equal to each other under different pressures along with the increasing speed. And for grinding with same pressure, the lower speed grinding spends more time in the same place with rotating wheel, thus resulting in energy surplus. The average depth of each passing at higher speed grinding is smaller than the lower speed, the repeated grinding passes will helps the higher-speedgrinding achieve the grinding assignments. The total grinding time consumed for the grinding (combining all the repeated passing) also

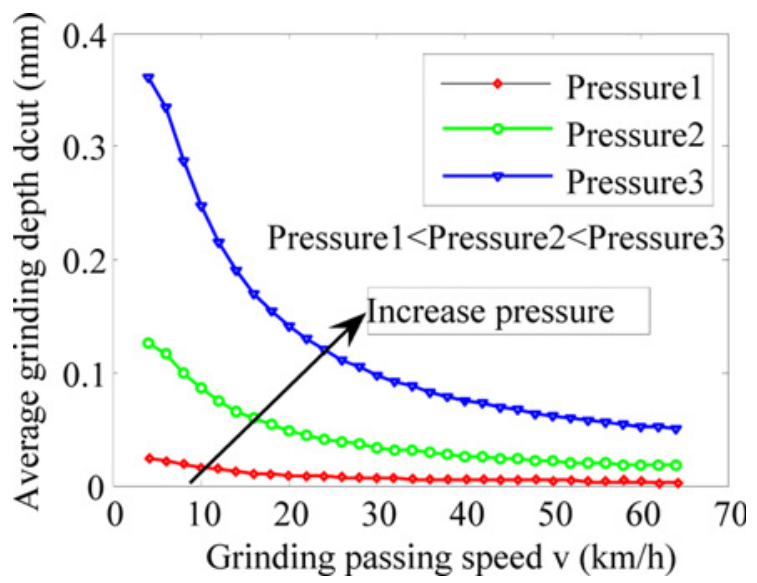

Fig. 21 Average grinding depth on different pressures

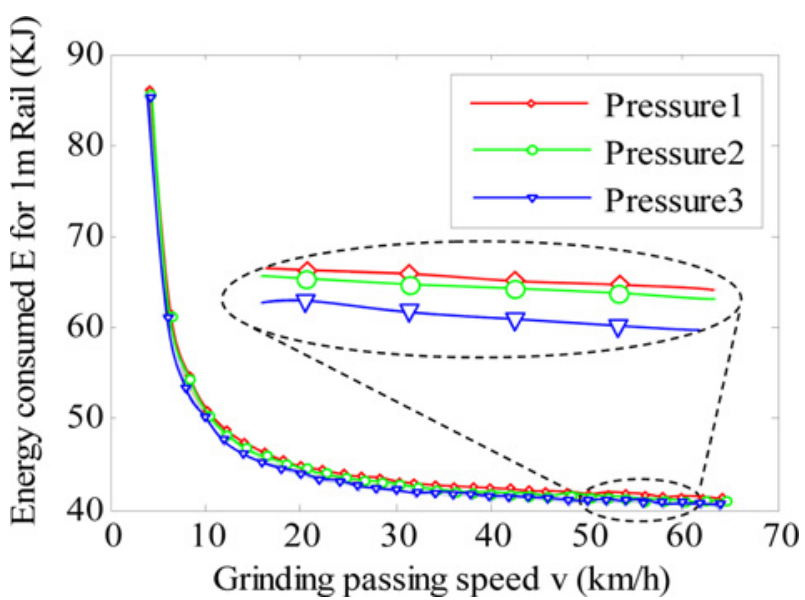

Fig. 22 Energy consumed on $1 \mathrm{~m}$ Rail for certain depth 
decreases along with the increasing of passing speed. Though the necessary repeat for the lower speed grinding are less than the higher speed, the overall time spent for finishing the same grinding assignments is still larger than the high-speed grinding, as shown in Fig. 23.

With same repeated times of passing, the higher pressure will cost more energy for $1 \mathrm{~m}$ rail as shown in Fig. 24. Higher speeds under same pressure will cost less energy for $1 \mathrm{~m}$ rail than lower speeds because of the fixed length of the workpiece. Therefore, it comes to a decision that a judgment for energy-saved grinding should depend on the grinding aims. If the grinding aim is to remove a shallow layer of metal within very limited grinding time, the energy-saved grinding would be the one with lower pressure and higher forward speed. For the greater removal aims without concerns over the grinding time (or the passing times), the energy-saved grinding method would be the one with lower pressure and lower speed (so as to control the power fluctuation on the rotating wheels for the sake of prolonging the service life of the grinding motor). But if the grinding passing times are limited for the sake of the economic benefits, higher pressures should be applied.

\section{Conclusions}

The rail grinding technology is environment-friendly, steel-saved and transit-energy-saved which needs necessary model for further

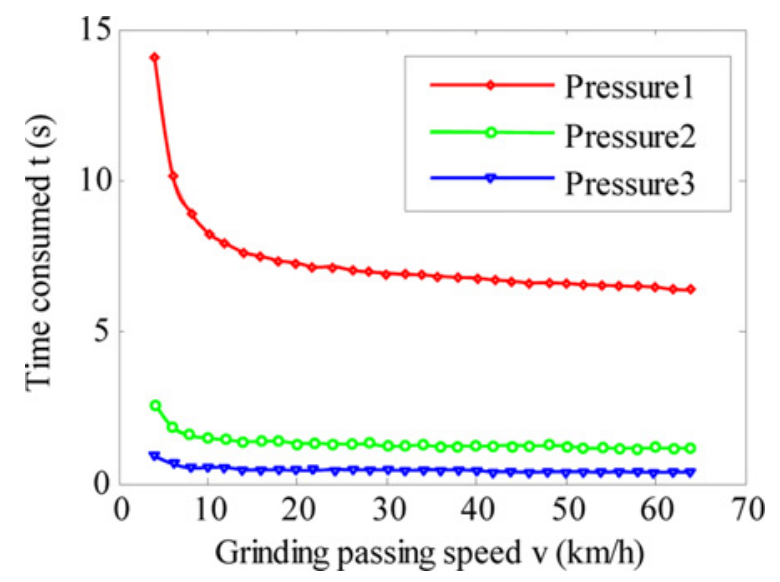

Fig. 23 Total time consumed under variable speeds

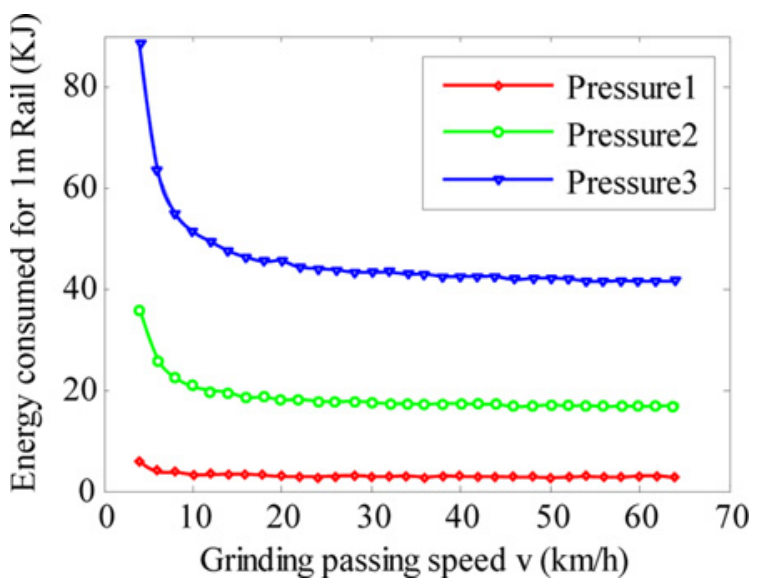

Fig. 24 Energy consumed with same repeated times investigation. As to focus on the grinding process and its consumed powers, the geometrical model of cutting grains, the cutting mechanism of the cutting grains and also the power-model have been built sequentially. The power-model has close relationship with the grain geometry, involved grain numbers and also the cutting speeds. The higher forward speed will bring along the lager fluctuation on the rotation powers and increase the powers spent on the traction. And increase the cutting depth will increase the numbers of involved grains and the consumed powers significantly. It then draws a conclusion that a judgment for energy-saved and motor-friendly grinding depends on the grinding aims. For shallow layer removed off the rail and limited working time, it will be suitable to choose lower pressure but higher forward grinding speed to accomplish the work. And for the requisite deep layer to be removed, it is suitable to operate the rail grinding at lower forward speed but with greater pressure.

\section{REFERENCES}

1. Dornfeld, D. A., "Moving towards Green and Sustainable Manufacturing," Int. J. Precis. Eng. Manuf.-Green Tech., Vol. 1, No. 1, pp. 63-66, 2014.

2. Choi, H.-Y., Lee, D.-H., Song, C. Y., and Lee, J., "Optimization of Rail Profile to Reduce Wear on Curved Track," Int. J. Precis. Eng. Manuf., Vol. 14, No. 4, pp. 619-625, 2013.

3. Zarembski, A. M., "High-Speed Rail Grinding and Metal Removal," Railway Track and Structures, Vol. 108, No. 6, 2012.

4. Zarembski, A. M., "The Art and Science of Rail Grinding," Simmons-Boardman Book, 2005.

5. Schoech, W., "New Rail Maintenance Trends in Europe AntiHeadcheck-Profiles and Preventive Cyclical Grinding," Proc. of the AusRAIL PLUS, 2009.

6. Malkin, S. and Guo, C., "Grinding Technology: Theory and Application of Machining with Abrasives,” Industrial Press Inc., 2008.

7. Darafon, A., Warkentin, A., and Bauer, R., "3D Metal Removal Simulation to Determine Uncut Chip Thickness, Contact Length, and Surface Finish in Grinding," The International Journal of Advanced Manufacturing Technology, Vol. 66, No. 9-12, pp. 17151724, 2013.

8. Cao, Y., Guan, J., Li, B., Chen, X., Yang, J., et al., "Modeling and Simulation of Grinding Surface Topography Considering Wheel Vibration," The International Journal of Advanced Manufacturing Technology, Vol. 66, No. 5-8, pp. 937-945, 2013.

9. Agarwal, S. and Rao, P. V., "Predictive Modeling of Force and Power based on a New Analytical Undeformed Chip Thickness Model in Ceramic Grinding," International Journal of Machine Tools and Manufacture, Vol. 65, pp. 68-78, 2013. 